\title{
Patients' Perception of Newly Initiated Oral Anticoagulant Treatment for Atrial Fibrillation: an Observational Study
}

\author{
Josiane Larochelle, M.Sc ${ }^{7}$, Caroline Brais, M.Sc ${ }^{2}$, Lucie Blais, Ph. D, ${ }^{3,4}$, Sylvie Perreault, Ph.D ${ }^{3}$, \\ Paul Farand, M. Sc, FRFCP ${ }^{5,6}$, Geneviève Letemplier, MD, FRFCP ${ }^{5,6}$, and Marie-France Beauchesne, \\ Pharm. D $3,4,5,6,7$
}

\begin{abstract}
'Institut universitaire de gériatrie de Sherbrooke, Sherbrooke, Québec, Canada; ${ }^{2}$ Hôpital du Haut-Richelieu, Saint-Jean-sur-Richelieu, Québec, Canada; ${ }^{3}$ Faculté de pharmacie, Université de Montréal, Montréal, Québec, Canada; ${ }^{4}$ Centre de recherche, Hôpital du Sacré-Cœur de Montréal, Montréal, Québec, Canada; ${ }^{5}$ Faculté de médecine et des sciences de la santé, Université de Sherbrooke, Sherbrooke, Québec, Canada; ${ }^{6}$ Centre hospitalier universitaire de Sherbrooke, Sherbrooke, Québec, Canada; ${ }^{7}$ Centre de recherche, Centre hospitalier universitaire de Sherbrooke, Sherbrooke, Québec, Canada.
\end{abstract}

KEY WORDS: atrial fibrillation; oral anticoagulants; warfarin; apixaban; dabigatran; rivaroxaban.

J Gen Intern Med 33(8):1239-41

DOI: $10.1007 / \mathrm{s} 11606-018-4457-z$

(C) Society of General Internal Medicine 2018

\section{INTRODUCTION}

Patient's satisfaction with oral anticoagulants (OAC) for the treatment of atrial fibrillation has been reported, ${ }^{1-4}$ but not in new users of warfarin or direct oral anticoagulants (DOACs). The main objective of this study was to describe patient's expectations and satisfaction with oral anticoagulant treatment.

\section{METHODS}

This prospective observational study recruited subjects with non-valvular atrial fibrillation or atrial flutter during hospital admissions between February 2013 and December 2014. The institutional scientific and ethics committees approved this study.

Patients were at least 18 years old, had a diagnosis of non-valvular atrial fibrillation or atrial flutter, were able to speak and read French or English, and were newly prescribed an $\mathrm{OAC}$ during admission that was continued at discharge (either warfarin or a DOAC [apixaban, dabigatran, or rivaroxaban]). Exclusion criteria were cognitive impairment, delirium, schizophrenia, OAC use for at least 1 month in the last year, moderate to severe mitral stenosis, heart valve replacement, congenital cardiac disease, uncontrolled hyperthyroidism, substance abuse, drug intoxication, an indication other than atrial fibrillation for the $\mathrm{OAC}$, atrial fibrillation episode of less than $48 \mathrm{~h}$, and peri- or post-operative atrial fibrillation.
Baseline characteristics were collected from medical charts and by patient interviews. To assess our primary outcome, expectations and satisfaction of patients towards their anticoagulant treatment were assessed using the "Perception of anticoagulant treatment questionnaire (PACT-Q)" 5,6 The PACT-Q1 includes 7 questions on patients' expectations. The PACT-Q2 includes 20 questions on treatment convenience $(n=11$ items), burden of disease and treatment ( $n=2$ items), and anticoagulant treatment satisfaction ( $n=7$ items) scored on a 5-point Likert-like scale. The PACT-Q1 is to be administered before treatment initiation, but because anticoagulation prescription was an eligibility criteria, it was administered during hospitalization as soon as possible following treatment initiation. The PACT-Q2 is to be administered once treatment is ongoing, and was mailed to the patients 3 and 6 months following discharge.

Mean scores for each of the seven items of the PACTQ1 were estimated in the warfarin and DOAC groups. For the PACT-Q2, two dimension scores were calculated, the convenience score including 13 items (combining convenience $[n=11$ items] and burden of disease dimensions $[n=2$ items $]$ ) and the satisfaction score (including 7 items). Convenience and burden of disease items were reversed (reversed score $=6$-item score), added together, and rescaled on a 0-100 scale to obtain a convenience dimension score. Satisfaction items were summed and rescaled on a $0-100$ scale to determine the satisfaction dimension score. Mean scores were compared between subjects on warfarin and subjects on DOACs using Student $t$ tests (IBM® SPSS ${ }^{\circledR}$ Statistics software, version 20).

\section{RESULTS}

Among 159 included subjects, 71 were prescribed warfarin and 88 a DOAC, mainly rivaroxaban (71.6\%)

Published online April 26, 2018 
Table 1 Baseline Characteristics

\begin{tabular}{|c|c|c|c|}
\hline Characteristics & Warfarin $(n=71)$ & DOAC $(n=88)$ & $p$ value \\
\hline Age (years), mean $\pm \mathrm{SD}$ & $72.8 \pm 10.8$ & $69.9 \pm 12.3$ & 0.12 \\
\hline Male gender, $n(\%)$ & $41(57.7)$ & $56(63.6)$ & 0.45 \\
\hline \multicolumn{4}{|l|}{ BMI $\left(\mathrm{kg} / \mathrm{m}^{2}\right), n(\%)$} \\
\hline$<18.5$ & $2(2.8)$ & 0 & \multirow[t]{4}{*}{0.22} \\
\hline $18.5-24.9$ & $13(18.3)$ & $24(27.3)$ & \\
\hline $25.0-29.9$ & $25(35.2)$ & $29(32.9)$ & \\
\hline$>30.0$ & $31(43.7)$ & $35(39.8)$ & \\
\hline \multicolumn{4}{|l|}{ Smoking status, $n(\%)$} \\
\hline Current smoker & $7(9.9)$ & $10(11.4)$ & \multirow[t]{3}{*}{0.35} \\
\hline Non smokers & $16(22.5)$ & $32(36.4)$ & \\
\hline Former smokers & $48(67.6)$ & $46(52.3)$ & \\
\hline \multicolumn{4}{|l|}{ Alcohol consumption, $n(\%)$} \\
\hline$<5$ drinks/week & $48(67.6)$ & $46(52.3)$ & \multirow[t]{4}{*}{0.44} \\
\hline 5-10 drinks/week & $6(8.5)$ & $11(12.5)$ & \\
\hline$>10 \mathrm{drinks} /$ week & $3(4.2)$ & $8(9.1)$ & \\
\hline Undetermined & $14(19.7)$ & $23(26.1)$ & \\
\hline Hospital length of stay, median; range (days) & $8 ;(1-29)$ & $5 ;(1-38)$ & 0.99 \\
\hline \multicolumn{4}{|l|}{ Unit of admission, $n(\%)$} \\
\hline Cardiology & $33(46.5)$ & $46(52.3)$ & \multirow[t]{6}{*}{0.01} \\
\hline Internal medicine & $18(25.4)$ & $15(17.0)$ & \\
\hline Emergency & $6(8.5)$ & $10(11.4)$ & \\
\hline Neurology & $2(2.8)$ & $6(6.8)$ & \\
\hline Pulmonary & $1(1.4)$ & $4(4.5)$ & \\
\hline Others & $11(15.5)$ & $7(8.0)$ & \\
\hline \multicolumn{4}{|l|}{ Liver enzymes } \\
\hline AST, median; range & $20 ; 8-107$ & $24 ; 13-319$ & 0.62 \\
\hline ALT, median; range & $20 ; 7-151$ & $20 ; 7-583$ & 0.91 \\
\hline eGFR, median; range $\left(\mathrm{mL} / \mathrm{min} / 1.73 \mathrm{~m}^{2}\right)$ & $65.2 ; 11.3-99.2$ & $76.8 ; 25.7-118.3$ & 0.004 \\
\hline $\mathrm{eGFR}<30 \mathrm{~mL} / \mathrm{min} / 1.73 \mathrm{~m}^{2}, n(\%)$ & $8(11.3)$ & $1(1.1)$ & 0.04 \\
\hline \multicolumn{4}{|l|}{ Diagnosis $n(\%)$} \\
\hline AF only & $49(69.0)$ & $64(72.7)$ & \multirow[t]{3}{*}{0.84} \\
\hline Atrial flutter only & $11(15.5)$ & $13(14.8)$ & \\
\hline $\mathrm{AF}$ and Atrial flutter & $11(15.5)$ & $11(12.5)$ & \\
\hline $\mathrm{CHADS}_{2} \geq 1, n(\%)$ & $54(76.1)$ & $63(71.6)$ & 0.001 \\
\hline $\mathrm{CHADS}_{2}$ score, mean $\pm \mathrm{SD}$ & $2.6 \pm 0.8$ & $1.9 \pm 1.3$ & 0.000 \\
\hline Heart failure, $n(\%)$ & $57(80.3)$ & $18(20.5)$ & 0.09 \\
\hline Hypertension, $n(\%)$ & $60(84.5)$ & $58(65.9)$ & 0.008 \\
\hline Diabetes, $n(\%)$ & $33(46.5)$ & $20(22.7)$ & 0.002 \\
\hline Stroke, $n(\%)$ & $8(11.3)$ & 17 (19.3) & 0.17 \\
\hline Coronary heart disease, $n(\%)$ & $40(56.3)$ & 34 (38.6) & 0.03 \\
\hline Peripheral vascular disease (other than stroke), $n(\%)$ & $15(21.1)$ & $9(10.2)$ & 0.06 \\
\hline History of falls, $n(\%)$ & $2(2.8)$ & $2(2.3)$ & 0.83 \\
\hline History of major bleeding, $n(\%)$ & $2(2.8)$ & $3(3.4)$ & 0.83 \\
\hline Number of drugs at discharge, median; range & $11 ; 1-30$ & $7.5 ; 2-18$ & 0.000 \\
\hline Polypharmacy (i.e., $\geq 5$ drugs/day) & $66(93.0)$ & $66(75.0)$ & 0.03 \\
\hline Concomitant ASA, $n(\%)$ & $25(35.2)$ & $13(14.8)$ & 0.007 \\
\hline Concomitant antiplatelets (other than ASA), $n(\%)$ & $14(19.7)$ & $2(2.3)$ & 0.001 \\
\hline
\end{tabular}

(Table 1). There were no significant differences in treatment expectations (PACT-Q1), with the exception of subjects on warfarin who had a slightly higher expectation of having side effects such as minor bruising or bleeding (Table 2, a). Convenience scores were similar in warfarin and DOAC users at 3 months and higher in the DOAC group at 6 months (Table 2, b). Satisfaction scores were similar at 3 and 6 months in both groups (Table 2, b).

\section{DISCUSSION}

Subjects on warfarin had a higher expectation of having minor side effects. In a review of studies assessing patients' perception towards the use of vitamin $\mathrm{K}$ oral antagonists (VKAs), it was found that the risk of bleeding was one of the factors which most concerned patients. ${ }^{4}$ Regarding convenience and treatment satisfaction, only convenience was higher in subjects on DOACs at 6 months, which is most likely due to not having to test INRs regularly. Fareau et al. also found that the convenience score was higher in subjects on DOACs. ${ }^{1}$ On the other hand, Fumigalli et al. reported that DOACs were associated with a higher degree of psychological satisfaction. ${ }^{2}$ Differences could be explained by the fact that subjects in our study have not experienced treatment with another OAC in the preceding year. This study has several limitations. The number 
Table 2 Results of the PACT-Q 1 Expectations and PACT-Q2 Convenience and Satisfaction

\begin{tabular}{|c|c|c|c|c|c|}
\hline \multicolumn{6}{|l|}{ A. PACT-Q1 expectations } \\
\hline Questions & \multicolumn{2}{|l|}{$\begin{array}{l}\text { Warfarin }(n=71) \text {, } \\
\text { average score, } \\
\text { mean } \pm \text { SD }\end{array}$} & $\begin{array}{l}\text { DOAC }(n=88), \\
\text { average score, } \\
\text { mean } \pm \text { SD }\end{array}$ & \multicolumn{2}{|l|}{$p$ value } \\
\hline $\begin{array}{l}\text { A1: Are you confident that your anticoagulation } \\
\text { therapy will prevent the formation of blood clots? }\end{array}$ & \multicolumn{2}{|l|}{$3.82 \pm 0.78$} & $3.81 \pm 0.76$ & \multicolumn{2}{|l|}{0.935} \\
\hline $\begin{array}{l}\text { A2: Do you expect that your anticoagulation therapy } \\
\text { will relieve some of your symptoms (such as pain } \\
\text { or swelling in your legs, palpitations, } \\
\text { breathlessness or chest pain, etc.)? }\end{array}$ & \multicolumn{2}{|l|}{$3.30 \pm 1.16$} & $3.07 \pm 1.36$ & \multicolumn{2}{|l|}{0.277} \\
\hline $\begin{array}{l}\text { A3: Do you expect your anticoagulation therapy to } \\
\text { have side effects such as minor bruising or } \\
\text { bleeding (for example, when shaving, cooking, } \\
\text { minor cuts, etc.)? }\end{array}$ & \multicolumn{2}{|l|}{$2.67 \pm 1.06$} & $2.29 \pm 1.00$ & \multicolumn{2}{|l|}{0.028} \\
\hline $\begin{array}{l}\text { A4: Is it important for you that your anticoagulation } \\
\text { therapy be easy to take? }\end{array}$ & \multicolumn{2}{|l|}{$3.86 \pm 0.95$} & $3.99 \pm 0.80$ & \multicolumn{2}{|l|}{0.359} \\
\hline $\begin{array}{l}\text { A5: Are you worried about making mistakes when } \\
\text { taking your anticoagulation therapy (for example, } \\
\text { how you take it, when you take it or the dose you } \\
\text { are taking)? }\end{array}$ & \multicolumn{2}{|l|}{$1.61 \pm 0.84$} & $1.50 \pm 0.90$ & \multicolumn{2}{|l|}{0.445} \\
\hline $\begin{array}{l}\text { A6: Is it important for you to be able to manage } \\
\text { your anticoagulation therapy yourself? }\end{array}$ & \multicolumn{2}{|l|}{$3.93 \pm 0.96$} & $3.85 \pm 1.10$ & \multicolumn{2}{|l|}{0.644} \\
\hline $\begin{array}{l}\text { A7: Are you worried about the money you may } \\
\text { have to spend for your anticoagulation therapy? }\end{array}$ & \multicolumn{2}{|l|}{$2.23 \pm 1.45$} & $2.06 \pm 1.37$ & \multicolumn{2}{|l|}{0.455} \\
\hline \multicolumn{6}{|c|}{ B. PACT-Q2 convenience and satisfaction (higher scores = convenient and satisfied) } \\
\hline \multirow[b]{2}{*}{ PACT-Q2 dimension scores } & & & \multirow{2}{*}{\multicolumn{2}{|c|}{$\begin{array}{l}6 \text { months } \\
\text { Average score, mean } \pm \mathrm{SD}\end{array}$}} & \\
\hline & SD & \multirow[t]{2}{*}{$p$ value } & & & $p$ value \\
\hline Warfarin $(n=52)$ & $\mathrm{DOAC}(n=54)$ & & Warfarin $(n=38)$ & DOAC $(n=48)$ & \\
\hline Convenience score & $89.96 \pm 10.12$ & 0.069 & $86.29 \pm 12.76$ & $90.97 \pm 8.62$ & 0.012 \\
\hline Satisfaction score & $69.25 \pm 14.41$ & 0.821 & $61.37 \pm 17.18$ & $69.02 \pm 14.22$ & 0.323 \\
\hline
\end{tabular}

of patients was small and subjects on warfarin had more comorbidities. The PACT-Q questionnaire was completed by about 50 to $60 \%$ of patients at 6 months. In conclusion, expectations, convenience, and treatment satisfaction were generally similar between new users of warfarin and DOACs.

Acknowledgements: Contributors: The authors acknowledge MarieHélène Turgeon, Katy Lavoie, and Claudie Rodrigue for their contribution to data collection and analysis.

Corresponding Author: Marie-France Beauchesne, Pharm.D; Faculté de pharmacie, Université de Montréal, C.P. 6128, succ Centre-Ville, Montréal, Québec H3C 3J7, Canada (e-mail: mariefrance.beauchesne@umontreal.ca).

Funding Information BMS-Pfizer alliance, Boehringer-Ingelheim, and Bayer provided an unrestricted grant for this study.

\section{Compliance with Ethical Standards:}

The institutional scientific and ethics committees approved this study.

Prior Presentation: Complete results from this work were not presented at any conference.
Conflict of Interest: Authors state that they have no conflicts of interest outside the funding received for the conduct of this study.

\section{REFERENCES}

1. Fareau S, Baumstarck K, Farcet A, et al. Evaluation de la qualité de vie des patients âgés en fibrillation atriale sous anticoagulants au long cours: antivitamine $\mathrm{K}$ versus anticoagulants oraux directs. Ger Psychol Neurophsychiatr Vieil $2015 ; 13: 45-54$.

2. Fumagalli S, Cardini F, Roberts AT, et al. Psychological effects of treatment with new oral anticoagulants in elderly patients with atrial fibrillation: a preliminary report. Aging Clin Exp Res 2015; 27:99-102.

3. Hanon O, Chaussade E, Gueranger P, Gruson E, Bonan S, Gay A. Patient-Reported Treatment Satisfaction with Rivaroxaban for Stroke Prevention in Atrial Fibrillation. A French Observational Study, the SAFARI Study. PLoS ONE 11(12): e0166218. doi:https://doi.org/10.1371/journal. pone.0166218

4. MasDalmau G, Arderiu ES, Enfedaque Montes MB, Solà I, Pequeño Saco S, Coello PA. Patients' and physicians' perceptions and attitudes about oral anticoagulation and atrial fibrillation: a qualitative systematic review. BMC Fam Pract 2017; 18:1-11.

5. Prins MH, Marrel A, Carita P, et al. Multinational development of a questionnaire assessing patient satisfaction with anticoagulant treatment: the 'Perception of Anticoagulant Treatment Questionnaire' (PACT-Q). Health Qual Life Outcomes. 2009;7:9.

6. Prins MH, Guillemin I, Gilet $\mathbf{H}$, et al. Scoring and psychometric validation of the Perception of Anticoagulant Treatment Questionnaire (PACT-Q). Health Qual Life outcomes. 2009;7:30. 